\title{
Non-symmetric membership function for Fuzzy-based visual servoing onboard a UAV.
}

\author{
M. A. Olivares-Méndez* and P. Campoy and C. Martínez and I. F. Mondragón B. \\ Computer Vision Group, DISAM, Universidad Folitécnica de Madrid, \\ Madrid, Spain \\ *E-mail: migutelangel olivaresoupmes \\ wru!disam. upm.es/colibri
}

\begin{abstract}
This paper presents Fuzzy Logic controllers for a pan \& tilt vision platform onboard an Unmanned Aerial Vehicle. This implementation allows the UAV to follow objects in the environment by using Lucas-Kanade visual tracker, in spite of the aircraft vibrations, and the movements of the objects and the aircraft. The improvement of the present work is focused in the definition of non-symmetric membership functions for the Fuzzy controllers. This update has been tested in real flights with an ummanned helicopter of the Computer Vision Group at the UPM, with a very successfully results, attaining a considerable reduction of the error during the tracking tests.
\end{abstract}

Keywords: Unmanned Aerial Velucles, Non-Symmetric membership function, Fuzzy Control, Visual servoing, pan-tilt visual platform.

\section{Introduction}

The unmanned aerial vehicle (UAV) has made its way quickly and decisively to the forefront of aviation technology. Opportunities exist in a broadening number of fields for the application of UAV systems as the components of these systems become increasingly lighter and more powerful. UAVs provide a cheap, and safe alternative to manned systems and often provide a far greater magnitude of capability.

Visual sensing can provide a source of data for relative position estimation, situation awareness, and a UAV's interaction with the physical world. It, probably, represents a preferable technology for these purposes than either GPS or INS. In several UAV projects, computer vision plays the most important role in the environmental sensing accomplished by UAVs. Some applications of vision for UAVs include, obstacle avoidance ${ }^{1}$, the works of Rathinam et al. ${ }^{2}$ and $\mathrm{Campoy}^{3}$ for inspection and monitoring of oil-gas 
pipelines, roads, bridges, power generation grids, and other civilian tasks, the uses of natural Landmarks for Navigation and Safe Landing ${ }^{4}$, the works of Mondragón with an omnidirectional camera for attitude estimation of $\mathrm{UAV} \mathrm{s}^{5}$, among others.

A VTOL-UAV (Vertical Take Off and Landing-UAV) has more nonlinearities states that a fixed winds UAV, because it can change its position with a fast movements, and can remaind in hover position for a long time, been affected, just, by the environment perturbations. The objective of this work is to develop an active visual-based system for object tracking on realtime onboard the UAV modeling the non-linearities states of this kind of UAV. For tracking objects the well know Lucas-Kanade-Tomasi tracker has been used ${ }^{6},{ }^{7}$ This visual-based object tracking is implemented using a pan \& tilt vision platform, controlled by Fuzzy controllers. A non-symmetric membership functions implementation using the MOFS (Miguel Olivares? Fuzzy Software) have been used for the definition of the inputs and output variables of the Fuzzy controllers, in order to fit better to the high non-linear system and improving the results of previous works ${ }^{8}, 9$

This is the principal reason for what the non-symmetric membership functions are implemented for this work,

For a better comprehension, the paper is divided in the following sections. In Section 2 we show a description about the UAV used in this work. Section 3 will show the Miguel Olivares' Fuzzy Software definition and the configuration of the different fuzzy controllers. The presentation of some results obtained for this work are shown in the Section 4 and finally, we present the conclusions and future works in Section 5.

\section{UAV System Description}

The Colibri project has three totally operative UAV platforms. One electric helicopter, and two gas powered helicopters. The COLIBRI testbeds, ${ }^{3}$ are equipped with an xscale-based flight computer augmented with sensors (GPS, IMU, Magnetometer, fused with a Kalman filter for state estimation). For this work, the UAV system used was an electric SR 20 of "Rotomotion", shown in Fig. 1.

It includes a two axis pan-tilt video-platform powered by two servomotors, with an action range of 180 degrees. For the control of the platform we send position commands to the servos through the helicopter controller, based on the visual information acquired. To perform vision processing, it has a VIA nano-ITX $1.5 \mathrm{GHz}$ onboard computer with 1 Gb RAM-DDR2. 


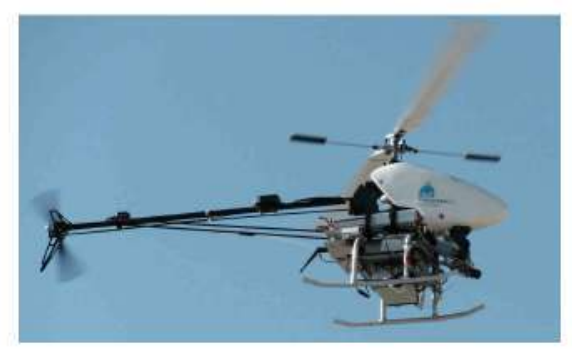

Fig. 1. COLIBRI III Electric helicopter.

\section{Visual servoing using Fuzzy controllers}

\subsection{Fuzzy Software Implementation}

The MOFS has been designed by the definition of one class for each part of the fuzzy-logic environment (variables, rules, membership functions and defuzzification modes) in order to facilitates the future updates and makes easier work with it. Actually, 3 different kind of membership function have been implemented, those are the pyramidal, trapezoidal and gaussian. Furthermore, the two inference models for the fuzzification that have been implemented are the maximum and product, also for the defuzzification is used the Height weight method. More details about the structure of this software is possible see at the. ${ }^{10}$

There are some difference between this fuzzy software and others. One is the learning algorithm based on the idea of the synaptic weights of the neurons, but for this work this improvement is not used, in ${ }^{11},{ }^{12}$ is possible to see how it works. The other improvement of this software is the possibility to define non-symmetric membership function for each fuzzy variables. This idea consist in the definition of different sizes for the different parts of the membership function as is shown in the central parts of the figures 2. With this improvement the system has a more adaptive response to the nonlinear model of this system that represent the helicopter movements and the different perturbations of the environment that can affect to the UAV. It must be considered that the helicopter can change it position with fast movements (more than $0.4 \mathrm{~m} / \mathrm{s}$ ) or slow movements (less than $0.4 \mathrm{~m} / \mathrm{s}$ ) in for there displacements ( $\mathrm{x}, \mathrm{y}$, and $\mathrm{z}$ ) and one rotation (the heading). Also, can remaind in hover position for a long time, been affected, its position, just for the environment perturbations. Some of these different changes of the helicopter will be explain with figures in the next sub-section.

The definition of the different sub-sets of each variables is based on the 
result of more than 20 different test. Those test have been made in the laboratory and onboard the UAV in real flights.

\subsection{Fuzzy Controllers}

To develop the visual servoing task in a vision pan \& tilt platform on board the UAV, two fuzzy controllers have been implemented using the MOFS. They are working in parallel, one for the pan axis of the vision platform and the other one for the tilt axis. The purpose of this implementation is to keep in the center of the image the tracking object, in spite of the helicopter vibrations and the movements of the object and the UAV. The two controllers are design using triangular membership function, the product inference model for the fuzzification and the Height Weight operation method for the defuzzification of the output (Eq. 1).

$$
y=\frac{\sum_{l=1}^{M} \bar{y}^{l} \prod\left(\mu_{B^{\prime}}\left(\bar{y}^{l}\right)\right)}{\sum_{l=1}^{M} \prod\left(\mu_{B^{\prime}}\left(\bar{y}^{l}\right)\right)}
$$

In Fig. 2(a) and 2(b) are shown the first input of each controller and in Fig. 2(c) the second input of them. The Fig. 2(d) shows the definition of the output for each controller. In those figures, it is possible to notice the definition of the non-symmetric membership functions.

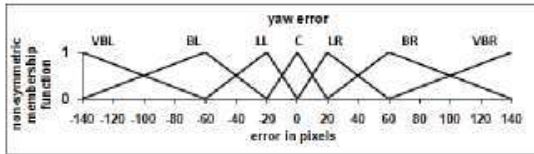

(a) First input variable of the Yaw controller.

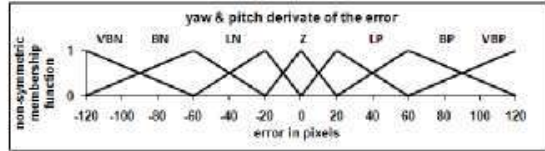

(c) Second input of the Yaw and Pitch Controllers.

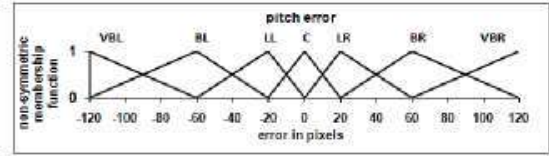

(b) First input Variable of the Pitch controller.

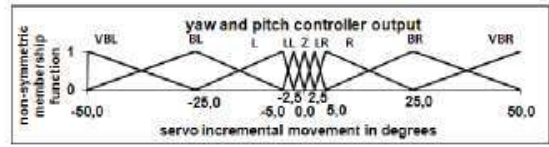

(d) Output of the Yaw and Pitch Controllers.

Fig. 2. Input and Output Variables for the Pitch and Yaw controllers.

\section{Experiments}

In this section we present a result of the performance of the two controllers using the new defined membership functions on a real test onboard the 
UAV. Here a real flight test were carried out. In figure 3, it is shown a 3D reconstruction of the flight using the GPS and the IMU data.

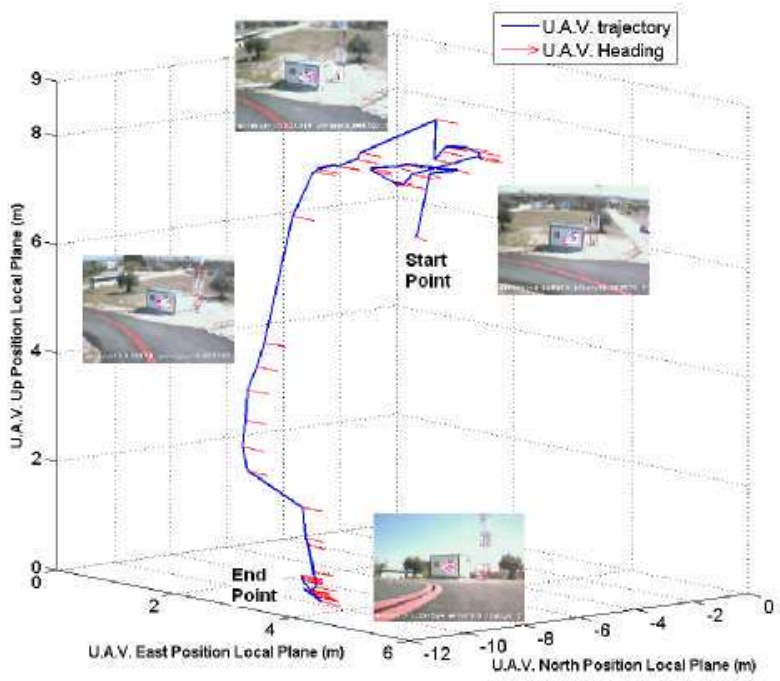

Fig. 3. 3D flight reconstruction using the GPS and the IMU data from the UAV. Where, the ' $\mathrm{X}$ ' axis represents the NORTH axis of the surface of the tangent of the earth, the ' $Y$ ' axis represents the EAST axis of the earth, the ' $Z$ ' is the altitude of the helicopter and the red arrows show the pitch angle of the helicopter.

In this figure, it is possible to see the trajectory of the UAV during the tracking task. In order to increase the difficult of the test, the selection of the object to track was made during the flight at the start point. At first, some rapid movements were made at the three axis (Figures 4 (a), 4 (b) and $4(\mathrm{c})$, before frame 180-200), making big changes to the different angles of the helicopter, as are shown in Figures $4(\mathrm{~d}), 4(\mathrm{e})$ and $4(\mathrm{f})$. Then a side movement with a increasing velocity was made (from $0.0 \mathrm{~m} / \mathrm{s}$ to more than $1 \mathrm{~m} / \mathrm{s}$ ), as is shown in Figure $4(\mathrm{c})$ from frame 180 to frame 300 . Later a rapid descend with a maximum of $1 \mathrm{~m} / \mathrm{s}$ was made, which is possible to see from frame 300 to frame 450 at the figure 4 (a) and $4(\mathrm{~d})$. The manual landing affected with big changes in all the angles when the helicopter was next to the floor. The test finalize with the vibrations of the helicopter when it was in contact with the terrain at the End Point.

This flight is faster than the presented in the previous works, in order to increase the difficult of the action to follow the tracked object.

In figure 5 , is possible to see the error in the two axis. Also, is possible 


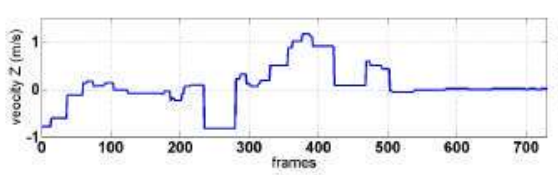

(a) Velocity changes in $\mathrm{Z}$ axis (altitude).

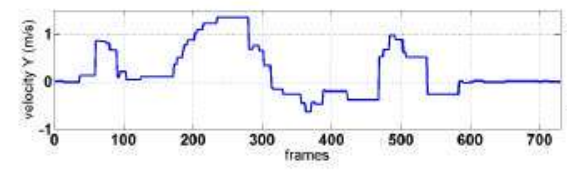

(c) Velocity changes in $\mathrm{Y}$ axis (Side).

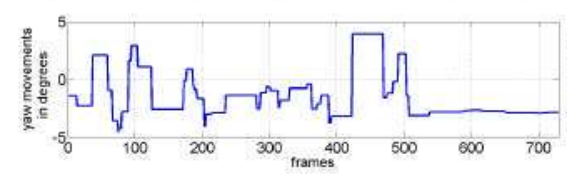

(e) Yaw angles changes.

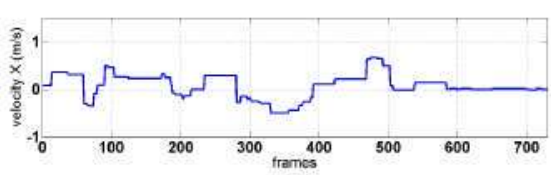

(b) Velocity changes in $\mathrm{X}$ axis (Forward).

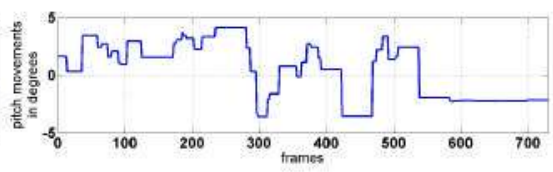

(d) Pitch angles changes.

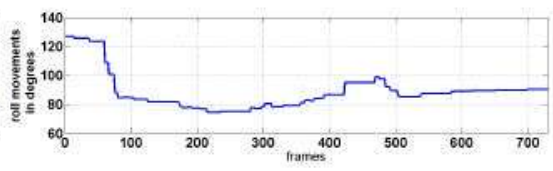

(f) Roll angles changes.

Fig. 4. Changes of the UAV in velocity and in degrees.

to see that there are not peak of error during the flight, obtaining a very good response of the controller. Furthermore, the results show the correct control tracking besides the increase of the movements, and the velocities in comparison with previous works. So, based in these results and the behavior of the controllers is possible to say that the improvement at the membership functions have a successful behavior to overcome the vibrations problems and the high non-linearity of the VTOL-UAV.

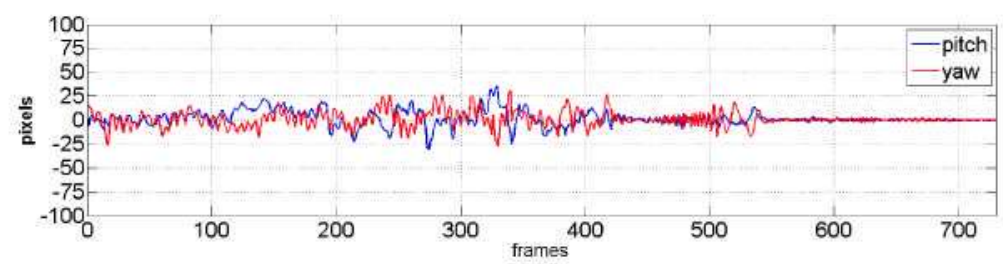

Fig. 5. Error between center of the image and center of the object to track.

\section{Conclusions and Future Works}

This work presents fuzzy controllers with non-symmetric membership functions on the definition of the input variables definition. These controllers 
are used for following objects by controlling a pan and tilt video platform on board a UAV. A Lucas-Kanade-Tomassi tracker is used. The controllers have a excellent behavior following the objects, obtaining better results than the previous results obtained without the non-symmetric membership function definition. The uses of the pan and tilt visual platform give to the helicopter a freedom of movements, as well as, a faster response when following moving objects, being the other implementations of visual servoing on UAV (without pan and tilt platform) more limited and slower than the platform servos response. The principal and immediate future work is to implement controllers for all the possible movements of the UAV (Forward, Side, altitude and heading) using velocity or position commands, in order to make a total control of the UAV and platform, in order to following objects without any restriction. It is possible to view the test videos and more on the web www.disam.upm.es/colibri.

\section{Acknowledgments}

This work is the product of several research stages at the Computer Vision Group Universidad Politécnica de Madrid. Has been sponsored by the Spanish Science and Technology Ministry under grants CICYT DPI2004-06624, CICYT DPI2000-1561-C02-02 and MICYT DPI2007-66156.

\section{References}

1. Z. He, R. Iyer and P. Chandler, Vision-based uav flight control and obstacle avoidance, in American Control Conference, 2006, June 2006.

2. S. Rathinam, Z. Kim, A. Soghikian and R. Sengupta, Vision based following of locally linear structures using an unmanned aerial vehicle, in Decision and Control, 2005 and 2005 European Control Conference. CDC-ECC' 05.44 th IEEE Conference on, Dec. 2005.

3. P. Campoy, J. Correa, I. Mondragon, C. Martinez, M. Olivares, L. Mejias and J. Artieda, Journal of Intelligent and Robotic Systems (2008).

4. A. Cesetti, E. Frontoni, A. Mancini, P. Zingaretti and S. Longhi, J. Intell. Robotics Syst. $\mathbf{5 7}, 233$ (2010).

5. I. F. Mondragón, P. Campoy, C. Martinez and M. Olivares, Robotics and Autonomous Systems In Press, Corrected Proof (2010).

6. B. D. Lucas and T. Kanade, An iterative image registration technique with an application to stereo vision (ijcaj), in Proceedings of the 7 th International Joint Conference on Artificial Intelligence (IJCAI' 81 ), April 1981.

7. C. Tomasi and T. Kanade, Detection and Tracking of Point Fentures, tech. rep., International Journal of Computer Vision (1991).

8. M. Olivares-Mendez, P. Campoy, C. Martinez and I. Mondragon, Eurofuse workshop 09, Preference modelling and decision analysis (Sept. 2009). 
9. M. Olivares-Mendez, P. Campoy, C. Martinez and I. Mondragon, A pan-tilt camera fuzzy vision controller on an unmanned aerial vehicle, in Intelligent Robots and Systems, 2009. IROS 2009. IEEE/RSJ International Conference on, Oct. 2009.

10. I. F. Mondragón, M. A. Olivares-Mendez, P. Campoy and C. Martinez, Autonomous Robots In Press, Corrected Proof (2010).

11. M. Olivares and J. Madrigal, Intelligent Signal Processing, 2007. WISP 2007. IEEE International Symposium on, 1(Oct. 2007).

12. M. Olivares, P. Campoy, J. Correa, C. Martinez and I. Mondragon, Fuzzy control system navigation using priority areas, in Proceedings of the 8th International FLINS Conference, (Madrid,Spain, 2008). 